\title{
Virtual surgical planning and 3D printing in prosthetic orbital reconstruction with percutaneous implants: a technical case report
}

This article was published in the following Dove Press journal:

International Medical Case Reports Journal

31 October 2016

Number of times this article has been viewed

\author{
Yu-Hui Huang',2 \\ Rosemary Seelaus ${ }^{1,2}$ \\ Linping Zhao ${ }^{1,2}$ \\ Pravin K Patel ${ }^{1,2}$ \\ Mimis Cohen ${ }^{1,2}$ \\ 'The Craniofacial Center, Department \\ of Surgery, Division of Plastic \& \\ Reconstructive Surgery, University \\ of Illinois Hospital \& Health Sciences \\ System, ${ }^{2}$ University of Illinois College \\ of Medicine at Chicago, Chicago, \\ IL, USA
}

\begin{abstract}
Osseointegrated titanium implants to the cranial skeleton for retention of facial prostheses have proven to be a reliable replacement for adhesive systems. However, improper placement of the implants can jeopardize prosthetic outcomes, and long-term success of an implant-retained prosthesis. Three-dimensional (3D) computer imaging, virtual planning, and $3 \mathrm{D}$ printing have become accepted components of the preoperative planning and design phase of treatment. Computer-aided design and computer-assisted manufacture that employ conebeam computed tomography data offer benefits to patient treatment by contributing to greater predictability and improved treatment efficiencies with more reliable outcomes in surgical and prosthetic reconstruction. 3D printing enables transfer of the virtual surgical plan to the operating room by fabrication of surgical guides. Previous studies have shown that accuracy improves considerably with guided implantation when compared to conventional template or freehand implant placement. This clinical case report demonstrates the use of a 3D technological pathway for preoperative virtual planning through prosthesis fabrication, utilizing 3D printing, for a patient with an acquired orbital defect that was restored with an implant-retained silicone orbital prosthesis.
\end{abstract}

Keywords: computer-assisted surgery, virtual surgical planning (VSP), 3D printing, orbital prosthetic reconstruction, craniofacial implants

\section{Introduction}

Percutaneous, osseointegrated, titanium implants in the cranial skeleton have demonstrated reliability for retention of facial prosthesis as an alternative to adhesive systems. Titanium implants are driven into bone, allowed to heal, and subsequently exposed through the skin to form secure anchorage for prosthetic retention. ${ }^{1,2}$ The successful aesthetic outcome of prosthetic rehabilitation relies upon precise and proper placement of implants in viable bone. . $^{3,4}$

The adoption of three-dimensional (3D) technologies has broadened the possibilities for surgical planning to ensure improved outcomes in craniofacial reconstruction. ${ }^{5}$ Virtual surgical planning (VSP), cone-beam computed tomography (CBCT), computer-aided design (CAD), and computer-assisted manufacture (CAM) have enabled improved and more precise planning to determine the necessary dimensions, positions, and angulations of implants. ${ }^{6,7}$ Virtually planned surgery can be executed efficiently by transfer of the plan to the operating theater by either navigation systems or the fabrication of surgical guides by 3D printing. ${ }^{89}$ Previous studies have shown that accuracy is considerably improved with guided implantation when compared to conventional template or freehand implant placement. ${ }^{10,11}$
Correspondence: Rosemary Seelaus Craniofacial Center, Department of Surgery, Division of Plastic and Reconstructive Surgery, University of Illinois Hospital \& Health Sciences System, 8 II South Paulina Street, Chicago, IL 606/2-73I4, USA

$\mathrm{Tel}+\mathrm{I} 3129967546$

Fax $+\mid$ 3I2 4I3 II57

Email rseelaus@gmail.com 
When employed, the materials and 3D printing process chosen for the fabrication of the surgical guide must produce a guide that is accurate, durable, and able to withstand sterilization. With their increased popularity, 3D printing technologies continue to improve in accuracy, material selection, faster printing times, and lower costs. ${ }^{12}$ Significant advances have been recently made toward this end; BioCell printing allows the manufacturing of $3 \mathrm{D}$ biodegradable and magnetic composite scaffolds made of poly( $\varepsilon$-caprolactone) and reinforced with iron-doped hydroxyapatite nanoparticles to improve bone tissue regeneration. ${ }^{13,14}$

This clinical case report demonstrates the application of $3 \mathrm{D}$ technologies for prosthetic rehabilitation of a patient with an orbital defect based upon virtually planned implant surgery, use of 3D-printed surgical guides, and CAM of a prosthetic prototype.

\section{Methods}

\section{Clinical report}

A 48-year-old woman with a right orbital defect who underwent exenteration and cranioplasty for fibromyosarcoma with a history of wearing an adhesive-retained orbital prosthesis was treated with surgical placement of implants in the right orbit, followed by fabrication of a custom, magnet-retained right oculo-orbital silicone prosthesis (Figure 1). Written informed consent was received by the patient for use and publication of these figures.

\section{Data acquisition}

CBCT scan data was captured for VSP (iCAT scanner, Next Generation 17-19, Imaging Sciences International, Hatfield, PA, USA). The scan was captured in 17.9 seconds with extended field of view $(23 \mathrm{~cm}$ diameter and $17 \mathrm{~cm}$ height). The voxel size measured $0.3 \times 0.3 \times 0.3 \mathrm{~mm}$. The

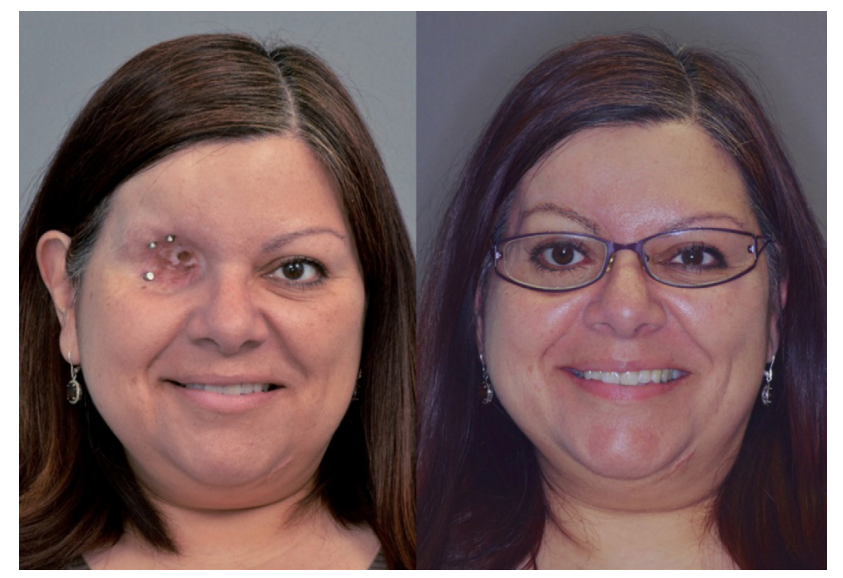

Figure I Pre- and post-prosthetic treatment photos of the patient fitted with an implant-retained orbital prosthesis.
CBCT data files (Digital Imaging and Communications in Medicine format) were imported and segmented to create the patient-specific virtual model that would be used for planning (Mimics Innovation Suite, Materialise, Leuven, Belgium).

\section{Virtual implant planning}

The *.stl files of the patient's bony anatomy and unaffected soft tissue orbit were generated. The soft tissue orbit, representing the future restoration was copied, mirrored, and positioned to simulate the best aesthetics for optimal symmetry. Based upon the planned restoration, implant positions were determined. Six CAD-generated virtual implants were positioned along the bony orbit according to surgical convention and prosthetic design considerations. The six implants were virtually placed in the $3 \mathrm{D}$ viewing window, perpendicular to the bone surface at the 1:00, 7:00, 8:00, 10:00, 11:00, and 12:00 o'clock positions per standard protocol (Mimics, Materialise). The coronal, axial, and sagittal views (Figure 2) were used to evaluate the available bone at the selected locations, and implant positions were adjusted as needed, taking into account the prosthetic design requirements. Angulation, length, and diameter were assessed to remain within the plane of the orbital rim for optimal prosthetic treatment.

\section{Surgical guide design}

The surgical guides were virtually designed and *.stl files were generated for printing (Mimics). The surgical guide was designed to be $3 \mathrm{~mm}$ thick and to incorporate areas of anatomical detail that would assist with positive registration on the bone surface. Five millimeter high access holes were designed at the implant sites to guide drilling for proper angulation. Boolean subtraction was used to define the access holes for drill guidance. A Boolean operation was also used to create the bone fitting surface of the guide (Figure 3 ). The final design was then exported as an *.stl file for printing.

\section{Surgical guide manufacture}

The surgical guide was printed on an uPrint SE Plus 3D Printer (Stratasys, Eden Prairie, MN, USA) with a layer thickness of $0.253 \mathrm{~mm}$ in acrylonitrile butadiene styrene plastic. Supporting structures were dissolved with the WaveWash support cleaning system (Stratasys).

\section{Patient's skull reference model}

To serve as a reference for the fit of the surgical guide, a 3D model of the patient's partial skull was printed from the *.stl data of the patient's skeletal anatomy (ZPrinter ${ }^{\circledR} 310$ Plus [Z Corp, Cambridge, MA, USA]). The model was printed with a layer thickness of $0.102 \mathrm{~mm}$ in high-performance 


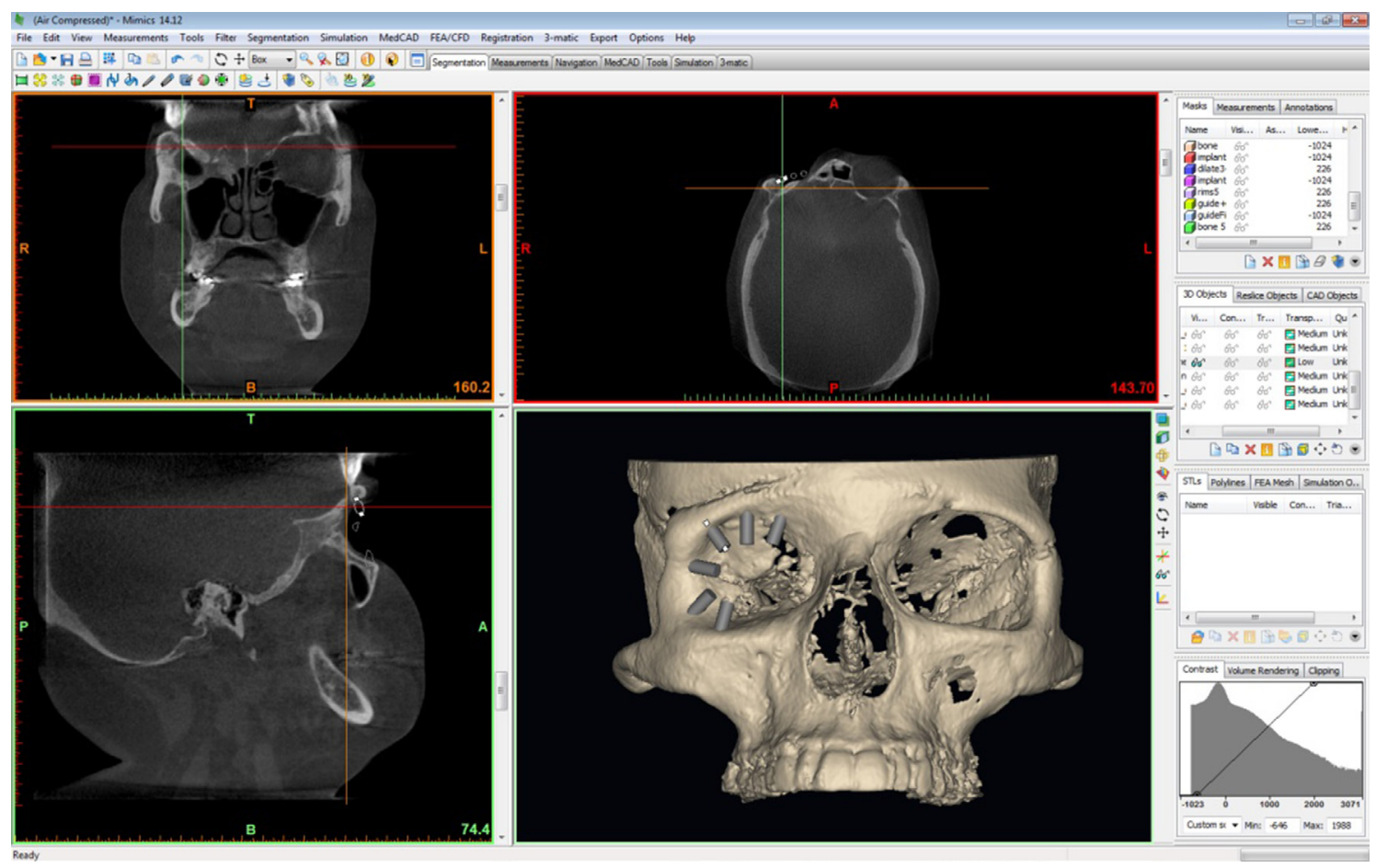

Figure 2 The views in Mimics, including coronal, axial, sagittal, and three-dimensional reconstruction.



Figure 3 Virtual (left) and physical (right) computer-generated models of the patient's skull with surgical guide and implants.

composite powder and binder to solidify the layers. The printed model was infiltrated with cyanoacrylate to seal the surface area and provide strength.

\section{Evaluation: fitting test and simulation}

The surgical guide was test fitted on the 3D-printed model of the patient's anatomy to ensure a precise fit in the area for surgical placement of the implants (Figure 3). The locations of the drilling sites were assessed by clinicians to ensure that they adequately represented the surgical plan before proceeding to surgery. The surgical guide and partial skull were then sterilized for surgery (Sterrad ${ }^{\circledR} \mathrm{NX}^{\circledR}$ System, ASP, Irvine, CA, USA).

\section{Stage I surgery - orbital implant}

With subperiosteal exposure of the orbital bone, the surgical guide was seated directly onto the orbital rim without the need for external fixation (Figure 4$)$. The implant positions of 7:00, 8:00, 10:00, 11:00, and 12:00 o'clock positions were determined to be optimal. A marking drill was used to mark the position and angulation of each implant through the access holes of the surgical guide. Four $3 \mathrm{~mm}(3.75 \mathrm{~mm}$ diameter) and one $4 \mathrm{~mm}$ long craniofacial implants (Vistafix ${ }^{\circledR}$ Prior Generation, Cochlear ${ }^{\mathrm{TM}}$, Sydney, NSW, Australia) were placed in the designated implant locations. Cover screws were placed and the surgical site was closed in customary fashion.

\section{Stage 2 surgery - abutment connection and orbital prosthesis fabrication}

A second surgery was performed 3.5 months later for abutment connection. Three implants were connected at the 8:00, 11:00, and 12:00 o'clock positions, and $7.5 \mathrm{~mm}$ healing abutments were attached (Cochlear; Figure 4). The 7:00 and 10:00 o'clock positions were left as "sleeper" implants. Following healing, the definitive prosthesis was fabricated based upon a replica prototype printed from the virtual surgical plan. The prosthesis was finished in customary fashion and fitted with optimal prosthetic outcomes and patient satisfaction (Figure 1).

\section{Results}

The five implants that were placed in the orbital bone successfully osseointegrated. The three implants that were 


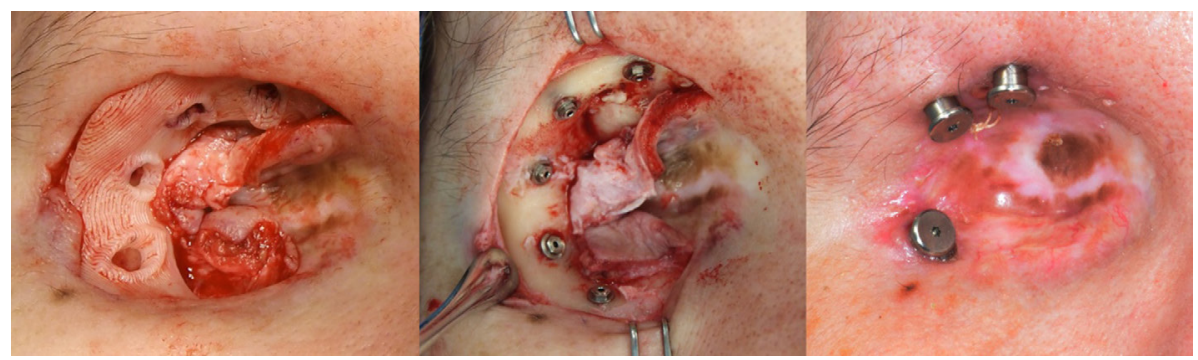

Figure 4 (Left to right) Surgical guide seated onto the orbital rim in situ for placement of five osseointegrated implants at 7:00, 8:00, 10:00, II:00, and I2:00 o'clock positions in the right orbit; followed by stage 2 abutment connection at 8:00, I1:00, and 12:00 o'clock positions.

connected with percutaneous abutments were stably connected and torqued to $20 \mathrm{NcM}$. Minimagnacap keepers (Minimagnacap S-Range, prior generation Vistafix) were easily connected to the abutments for prosthetic retention. Periabutment tissue healed well and remained stable without incident. The patient was easily able to place and remove the orbital prosthesis with magnet retention, and reported reliable retention.

\section{Discussion}

Computer-assisted technologies and 3D printing have become an accepted standard of care for preoperative planning and prosthesis design. 3D modeling and simulation software allows virtual reconstruction of the craniofacial structure and enables simulation of the surgical procedure to plan an optimal postoperative result. Modeling and simulation software provides clinicians the ability to work interactively with patient-specific virtual models to simulate surgical procedures and improve the planning process. The fabrication of the custom-fitting surgical guide serves as the "bridge" between virtual implant planning and surgery.

In the present study, the surgical guide was fabricated and assessed by clinicians on a 3D-printed model of the patient's skeletal anatomy. The guide when placed on the intended anatomical site resisted movement, indicating a precise fit between the guide and the bone surface. No distortion problems were experienced with sterilization (Sterrad NX), or with usage of the guide intraoperatively. The guide reduced the time spent in the operating room and enabled the surgery to be performed with greater accuracy, contributing to the optimal outcome for the patient. We believe the time and cost investment for planning and guide production compared favorably, justifying this preoperative approach given the described benefits. A more detailed time and cost comparison would yield improved understanding of the overall cost-effectiveness of the use of surgical guides in craniofacial surgeries.
A $3 \mathrm{~mm}$ thick guide proved sufficiently durable for this case. Design improvements related to the bone fitting surface area could be identified and will be taken into consideration for future guide design. The surgical team considered the use of 3D virtual planning and printing technologies to offer the benefits of improved time and procedural efficiency in the OR with a more predictable postoperative and prosthetic outcome for this patient.

\section{Acknowledgment}

This work was partially funded by financial support from Face the Future Foundation. We thank Lindsay McHutchion for her assistance with the orbital prosthesis.

\section{Disclosure}

The authors report no conflicts of interest in this work.

\section{References}

1. Toljanic JA, Eckert SE, Roumanas E, et al. Osseointegrated craniofacial implants in the rehabilitation of orbital defects: an update of a retrospective experience in the United States. J Prosthet Dent. 2005;94(2):177-182.

2. Parel SM, Branemark PI, Tjellstrom A, Gion G. Osseointegration in maxillofacial prosthetics. Part II: extraoral applications. J Prosthet Dent. 1986;55(5):600-606.

3. Miles BA, Sinn DP, Gion GG. Experience with cranial implantbased prosthetic reconstruction. J Craniofac Surg. 2006;17(5): 889-897.

4. Visser A, Raghoebar GM, van Oort RP, Vissink A. Fate of implantretained craniofacial prostheses: life span and aftercare. Int J Oral Maxillofac Implants. 2008;23(1):89-98.

5. Zhao L, Patel PK, Cohen M. Application of virtual surgical planning with computer assisted design and manufacturing technology to cranio-maxillofacial surgery. Arch Plast Surg. 2012;39(4): 309-316.

6. Tardieu PB, Vrielinck L, Escolano E. Computer-assisted implant placement. A case report: treatment of the mandible. Int J Oral Maxillofac Implants. 2003;18(4):599-604.

7. Holst S, Blatz MB, Eitner S. Precision for computer-guided implant placement: using 3D planning software and fixed intraoral reference points. J Oral Maxillofac Surg. 2007;65(3):393-399.

8. Di Giacomo GA, Cury PR, de Araujo NS, Sendyk WR, Sendyk CL. Clinical application of stereolithographic surgical guides for implant placement: preliminary results. J Periodontol. 2005;76(4): 503-507. 
9. Nickenig HJ, Wichmann M, Hamel J, Schlegel KA, Eitner S. Evaluation of the difference in accuracy between implant placement by virtual planning data and surgical guide templates versus the conventional free-hand method - a combined in vivo - in vitro technique using cone-beam CT (Part II). J Craniomaxillofac Surg. 2010;38(7): 488-493.

10. Ersoy AE, Turkyilmaz I, Ozan O, McGlumphy EA. Reliability of implant placement with stereolithographic surgical guides generated from computed tomography: clinical data from 94 implants. J Periodontol. 2008;79(8):1339-1345.

11. Lal K, White GS, Morea DN, Wright RF. Use of stereolithographic templates for surgical and prosthodontic implant planning and placement. Part I. The concept. J Prosthodont. 2006;15(1):51-58.
12. Cohen A, Laviv A, Berman P, Nashef R, Abu-Tair J. Mandibular reconstruction using stereolithographic 3-dimensional printing modeling technology. Oral Surg Oral Med Oral Pathol Oral Radiol Endod. 2009;108(5):661-666.

13. Domingos M, Intranuovo F, Russo T, et al. The first systematic analysis of 3D rapid prototyped poly(epsilon-caprolactone) scaffolds manufactured through BioCell printing: the effect of pore size and geometry on compressive mechanical behaviour and in vitro hMSC viability. Biofabrication. 2013;5(4):045004.

14. De Santis R, Russo A, Gloria A, et al. Towards the design of 3D fiberdeposited poly(epsilon-caprolactone)/iron-doped hydroxylapatite nanocomposite magnetic scaffolds for bone regeneration. $J$ Biomed Nanotechnol. 2015;11(7):1236-1246.

\section{Publish your work in this journal}

The International Medical Case Reports Journal is an international peer-reviewed open-access journal publishing original case reports from all medical specialties. Previously unpublished medical posters are also accepted relating to any area of clinical or preclinical science. Submissions should not normally exceed 2,000 words or
Submit your manuscript here: https://www.dovepress.com/international-medical-case-reports-journal-journal 